Die Milch enthielt vor dem Schütteln: $5,0632 \mathrm{~g}$ Milchzucker in $100 \mathrm{ccm} ; \mathrm{n}$ ach dem Schütteln und Filtrieren fand sich $3,6019 \mathrm{~g}$ in $100 \mathrm{ccm}$.

Wir haben also 0,1406 Millimol pro Kubikzentimeter vor der Adsorption, 0,1000 Millimol im Kubikzentimeter $\mathrm{nach}$ der Adsorption. Es wurden aus $1 \mathrm{ccm}$ Milch 0,0406 Millimol von $4,9662 \mathrm{~g}$ Kohle adsorbiert. Die von $1 \mathrm{~g}$ Blutkohle überhaupt adsorbierte Menge $\left(\frac{x}{m}\right.$ beob.) beträgt 0,8175 Millimol. Die Formel: $\frac{x}{m}$ $=1,243 \mathrm{c}^{0,0823}$ (vgl. Tab. 5, Seite 129) liefert für die Gleichgewichtskonzentration 0,1000 den Wert 1,028. Die Adsorption in Milch ist für Milchzucker geringer als in wässeriger Lösung, und $z w a r$ wurde etwa $1 / 3$ der Milchzuckermenge bei unserem Versuche weniger adsorbiert. Dies beweist, daB die zahlreichen anderen Grenzflachen in der Milch sich auch an der Adsorption beteiligen. Immerhin findet, und das ist bemerkenswert, auch in Milch eine deutliche Adsorptiondes Milch. zuckers durch einefeste Grenzflache wie Kohle statt.

Resultate der Untersuchung.

1. Es wurden die Adsorptionen von Milchund Rohrzucker in wässeriger Lösung durch Blutkohle untersucht und die Adsorptionsisothermen aufgenommen.

2. Milch- und Rohrzucker werden ungefähr gleichstark durch Kohle adsorbiert, beide verhalten sich nicht wesentlich verschieden.
3. Auch in der Milch findet Adsorption des Milchzuckers durch Kohle statt, doch ist diese Adsorption merklich schwächer als in rein wässeriger Lösung Die Milch als heterogenes System hat eben noch eine Reihe anderer Grenzflächen, die sich an der Adsorption beteiligen.

4. Die Ursache für die Adsorption in wässeriger Lösung ist jedenfalls in einer Erniedrigung der Grenzflächenspannung KohleLösung zu suchen. Obgleich an der Grenzfläche Lösung--Luft die Oberflăchenspannung erhöht wird, scheinen an anderen Grenzflächen Erniedrigungen aufzutreten.

5. Es wird gezeigt, daB andererseits qualitativ alle Bedingungen für das Zustandekommen einer Adsorption der Zucker an einer Grenzfläche erfüllt sind, wenn man die Grenzfläche als eine Zone veränderten Druckes betrachtet. Für eine quantitative Durcharbeitung fehlt leider die Kenntnis der genauen Verhaltnisse.

6. Da die allerdings nicht prüfbare Annahme einer Erniedrigung der Oberflächenspannung an der Grenze Kohle-Lösung die Erscheinung quantitativ zu studieren gestattet und dieser Annahme, wie gezeigt wurde, keine ernstlichen Schwierigkeiten entgegenstehen, ist es geraten, dieselbe für die Zucker beizubehalten, solange die "Druckhypothese" keine bessere quantitative Durcharbeitung ermöglicht ${ }^{32}$ ).

82) H. Freundlich, Kapillarchemie (Leipzig 909), 289.

\title{
Zur Frage der Untersuchungsmethoden kapillarchemischer Probleme.
}

Von P. P. von Weim arn, St. Petersburg.

(Eingegangen am 29. Nov. 1910)

Vorbericht.

Beim Lesen des Novemberheftes der Koll.Zeitschr, war ich nicht wenig über den Inhalt des Artikels mon P. N. Pawlow erstaunt. Zwecks Orientierung der Leser sehe ich mich zu folgendem Vorbericht veranlaBt.

In den Punkten 1, 2, 3 kritisiert P. N. Pawlow die Frage von der Unmöglichkeit, die Formel von Clapeyron-Clausius zugleich mit der Formel von $L$ a place beim Schmelzprozeß hochdisperser Kristalle anzuwenden. wobei er seine Ausführungen derart abfaßt. daB der Leser, der mit dem Inhalt meines russischen, die ursprüngliche Arbeit von $P$. N. Pawlow kritisch beleuchtenden Artikels?) nicht vertraut ist, $z u$ der Ueberzeugung gelangen muB, als ob ich die Vergleichung der oben erwähnten Formeln nicht zum Beweise des falschen Weges, den P. N. Pawlow in seiner Arbeit eingeschlagen, sondern voll-

1) P. N. Pawlow: „Abhangigkeit der Schmelz. temperatur von der Oberflächenenergie eines festent Körpers* 40, 1022-1067 (1908).

2) P. P. v on Weimarn: "Physikalische und chemische Eigenschaften als Funktionen der Korngröße lester und flussiger disperser Phasen*, Joum. d. russ. chem. Ges. 40,1434 1447 (1908). 
kommen selbständig vorgenommen hätte. Da die Mehrzahl der Leser die russische Sprache nicht beherrscht und deshalb den lnhalt der Artikel sowohl von P. N. Paw low als auch von mir nicht kennt, so will ich so kurz als möglich dieselben rekapitulieren.

Im Septemberheft des Journ. d. russ. phys.chem. Ges. leitet P. N. Pawlow in der Abhandlung "Abhängigkeit der Schmelztemperatur von der Oberflachenenergie cines festen Körpers*, indem er annimmt, dab die Kornchen sich nur unter dem Druck des eigenen Dampfes befinden, aus der Gleichung

$$
\frac{d p}{p}=-\frac{d r}{r^{2}} \cdot \frac{2 k}{d R \sigma T}-\frac{d T}{T^{2}} \cdot \frac{1}{r} \cdot \frac{2 k}{d R \sigma}
$$

und aus der Gleichung Clapeyron-Clausius

$$
\rho=A T\left(v_{2}-v_{1}\right) \frac{d p}{d T}
$$

die Formel her:

$$
\frac{d T}{d r}=\frac{A\left(v_{2}-v_{1}\right) \frac{p}{r^{2}} \cdot \frac{2 K}{d R \sigma}}{\rho+A\left(v_{2}-v_{1}\right) \frac{p}{r} \cdot \frac{2 k}{d R \sigma T}}
$$

In der Voraussetzung, $\mathrm{daB}$ die latente Schmelzwärme o eine negative (1) Gröbe ist. wendel P. N. Pawlow diese Formel bei realen Schmelzfällen an und kommt zum Resultat, dab, je kle iuerdas Eiskörnchen (und ihm ähnliche Körper), um so höher die Schmelztemperatur ist; für gewöhnliche Substanzen (die mit einer Volumzunahme schmelzen) gelangt P. N. Pa w low zum umgekehrten Ergebnis, wie heim Eise (S. 1026 bis 1027).

3) Damit der Leser ersieht, welche Bedeutung P. N. Paw low den Folgerungen aus seiner Formel (3) beimibt, führe ich eine wörtliche Uebersetzung der entsprechenden Stellen aus seiner Abhandlung an (S. 1027 und 1061): ... für Substanzen, die sich durch Volumverringerung beim Uebergange aus dem festen in den flussigen $Z u$ stand charakterisieren, erhoht sich die 5 chmelztemperatur entsprechend der GroGenabnahme des schmelzenden Kornes und umgekehrt. . . Dieser Fall bietet ein besonderes Interesse dank dem Umstande, dab auch Wasser zur Zahl derjenigen Substanzen gehött, die die erwahnten Volumveränderungen erleiden ... Diese Kategorie von Körpern kann im Gegensatz zur ersten (für welche $v_{2}-v_{1}>0$ ) im uberhitzten Zustande nur kleine Körnchen haben .... im Schmelzgebiet (im iberhitzten Zustande) kann eine Flissigkeit durch mechanische Zerstaubung in einen festen Zustand ubergefuhrt werden." (Der gesperte Druck stammt von P. N. $\mathrm{Pa}$ w low.) Mag der Leser jetzt selbst diese Theorie von P. N. Pawlow mit der Theorie des Einflusses ungleichförmigen Druckes, von der er im Novemberheft der Koll. Z Zeitschr. spricht, miteinander vergleichen.
Als Antwort auf die Abhandlung von P. N. Pawlow verfaßte ich eine Entgegnung, die unter der Ueberschrift "Physikalische und chemische Eigenschaften als Funktionen der Korngröbe fester und flüssiger disperser Phasen" gedruckt wurde. Ich wies unter anderem auf folgendes hin: Ohne bei der durchaus nicht einwandfreien Ausführung der Formeln 1 und 3 von P. N. Paw low zu verweilen, zeigte ich, nachdem ich die absurde Voraussetzung von P. N. Pawlow - $\rho<0$ zurechtgestellt, dah die zusammengesetzte Formel 3 zum gleichen $\mathrm{Er}$ gebnis in betreff des Einflusses des Dispersitätsgrades auf das Schmelzen fester Körnchen führt, wie auch die einfache Betrachtung der Laplace'schen Formel vom Standpunkt des gewöhnlichen Einflusses des äußeren Druckes auf die Schmelztemperatur, wobei ich kategorisch darauf hinwies, dab ich persönlich in derartigen Fragen stets von molekularkinetischen Voraussetzungen a u s g e ga ng en bin, weil die Lehre von der Oberflächenschicht usw. zu Resultaten führt, die nicht mit der Erfahrung übereinstimmen (das geht deutlich aus der von mir kritisierten Theorie von P. N. Pawlow hervor). In Anbetracht dessen, daB P. N. Pawlow im Novemberheft in seiner Abhandlung diesen Punkt meiner Arbeit vollständig vorurteilsvoll ausgelegt hat, führe ich ihn hier vollständig an (S. 1443-1445): „Ich wende mich jetzt zu den auf Grund der Lehre von der Oberflächenenergie gemachten theoretischen Ausführungen der Abhängigkeit der Schmelztemperatur von der Korngröße. Vor allen Dingen muß ich darauf hinweisen, $d a \beta$ ich bei meinen Untersuchungen stets von molehular-kinetischen Vorstellungen ausgegangen bin, denn die auf Grund der Lehre von der Oberflächenenergie gezogenen Schlubfolgerungen ergeben, meiner Meinung nach, der Erfahrung direkt entgegengesetzte Resultate. So erhalt man z. B. für die Abhängigkeit der Schmelztemperatur von der KorngröBe, wie man aus meiner weiter unten folgenden Ausführung ersieht, ein dem experimenteilen entgegengesetztes Resultat ${ }^{*} . .{ }^{4}$.

„P. N. Pawlow erhielt ein umgekehrtes Resultat, was meiner Meinung nach, ein Mißverständnis darstellt. Nehmen wir die Gleichung von P. N. Pawlow:

) Folgende Zeilen stehe Koll.-Zeitschr. 6, 537 


$$
\frac{d T}{d r}=\frac{A \cdot\left(v_{2}-v_{1}\right) \frac{p}{r} \cdot \frac{2 K}{d R \sigma}}{\rho+A\left(v_{2}-v_{1}\right) \cdot \frac{p}{r} \cdot \frac{2_{K}}{d \cdot R \sigma T}}
$$

Er schreibt: „Da $\varrho<0$, die (übrigen) GröBen aber, alle positiv sind ..."(S. 1026). Zum ersten Male habe ich Gelegenheit zu lesen, daß die latente Schmelzwärme für reale Körper eine negative GroBe darstellt. Nehmen wir dagegen an, wie üblich, $\varphi>0, v_{g}-v_{1}$ aber $<0$, so erhalten wir ida $A\left[v_{2}-v_{1}\right] \cdot \frac{p}{r} \cdot \frac{2 K}{d R \sigma T}$ im Vergleich zu $\rho$ klein ist) $\frac{d T}{d r}>0$; d. h. bei den Körpern in der Art des Eises werden die allerkleinsten Kornchen leichtschmelzbarer; bei $v_{2}-v_{1}>0$

$$
\frac{d T}{d r}<0
$$

d. h. für die Mehrzahl der Körper steigt mit der Abnahme der Korngröbe die Schmelztemperatur. Auf diese Weise ergibt, indem man $\rho>0$ annimmt, die Formel von P.N. P a w low dasselbe Resultat, wie die meinige. Diese Resultate widersprechen aber der Erfahrung. Vom molekular-kinetischen Standpunkt aus, von dem ich in meinen Arbeiten ausgegangen bin, ist es vollkommen klar, daß die Schmelatemperatur für die kleinsten Körnchen eines beliebigen Körpers niedriger sein muß". (S. 1443-1445.)

Aus diesem Abschnitt geht deutlich hervor, daB die Theorie von P. N. Pawlow zu denselben Resultaten führt, wie auch die einfache Prüfung der Formel von Laplace vom Stanupunkt des .gewöhnlichen Einflusses des äuBeren Druckes auf die Schmelztemperatur ${ }^{6}$ ). Das ist auch leicht verständlich, denn der Weg von P. N. Pawlow hat außer der Kompliziertheit keinen Vorzug vor dem angeführten ${ }^{7}$ ). Hieraus folgt, daB die Punkte $1,2,3$ des Pawlow'schen Artikels im Novemberheft vor

5) Beilaufig bemerke ich, daB die Mehrzahi der im rechten Teil des Ausorucks befindlichen GroßBen ebenfalls Funktionen der KorngroBe sind.

) Obwohl P. N. Pawlow den von mir bezeichneten Fehler teilweise zugibt, sagt er doch, dab seine Folgerungen sich aut einen speziellen Fall des $\mathrm{Zu}$ standes des Korpers - wenn $\varphi<0-$ beziehen. (Dessenungeachtet wandte P. N. Pawlow diesen hypotetischen Fall aut das reale Schmelzen des Eises u.s. Korper an.) Journ. d. russ. chem. Ges. 41, 1779 (1909).

7) Eine weitere Theoric vun P. N. Paw low (Zeitsch. $t$. physik Chem. 65, 545, 1909) erscheint mir auch ganz unangemessen. allen Dingen auf P. N. Pawlow selbst bezogen werden müssen.

\section{A. Der Einflub des ungleichformigen Druckes.}

Auf S. 666 ${ }^{\circ}$ ) der "Theoretischen Chemie* von W. Nernst findet sich folgender Satz: „Der Schmelzpunkt gepreBten Eises (und jedes anderen festen Körpers), das mit der nicht gepreBten Schmelze in Berührung ist, muB durch auberen Druck in leicht berechenbarer Weise sinken." Da man auBerdem in betreff des Dampfdruckes, der Löslichkeit usw. bei ungleichförmigem Druck den allgemeinen SchluB im Sinne einer Zunahme aller dieser Eigenschaften ziehen kann, so ersieht man sofort ein Uebereinstimmen der Resultate bei den Versuchen mit den Eigenschaften hochdisperser Körper mit den Schlubfolgerungen der Theorie des Einflusses ungleichförmigen Druckes. Wem diese formale Uebereinstimmung von der Identität der Ursachen herzustammen scheint, dem steht augenscheinlich eine leichte Arbeit bevor, bestehend in der Umformung ( $\mathrm{mit}$ einigen entsprechenden Veränderungen) von H. Le Chatelie ${ }^{8}$ ) gegebenen Gleichungen; ich gehöre nicht $z u$ diesen, da ich die hier angeführte Theorie von Le Chatelier als eine "tote Theorie" betrachte, $d$. h. die sich auf die verschiedenen Naturerscheinungen nicht anwenden läBt. Diese Unanwendbarkeit ist nicht eine Folge von Fehlern in der Boweisführung, die von Le Chatelier mit der ihm eigenen gewöhnten Meisterschaft gegeben worden ist, sondern das Resultat der Zulassung der Möglichkeit, die eine Phase zu komprimieren, ohne mit der anderen dasselbe $z u$ tun. "Derartige Bedingungen" - schreibt Le Chatelier - finden sich verwirklicht, wenn man in einem Zylinder mittels eines nicht dicht schließenden Kolbens einen festen Körper in Stïcken, deren Zwischenräume von einer beweglichen Flüssigkeit erfüllt sind, zusammenpreBt, so daßs die Flüssigkeit aus dem Zylinder treten kann, ohne sich zusammendrücken zu lassen." Es ist leicht zu verstehen, daB ein solcher (oder ihm ähnlicher) Kolben zu gleicher Zeit die dünne Schicht der anderen Phase kom. primieren wird, die sich zwischen dem unteren Teil des Kolbens und der Oberfläche der durch den Kolben zusammengedrückten Phase befindet. Aus diesem Grunde werden die Ver-

9. 614 der Ausgabe 1900 .

9) Zeltschr. f. physik. Chem. 9, 335-338 (1892). 
anderungen des Systems nach der Theorie des gleichformigen Druckes erfolgen.

Alle zur Bestätigung der Theorie vom ungleichformigen Druck angeführten Beispiele ${ }^{10}$ ) werden vollkommen durch die Theorie des gleichformigen Druckes erklärt, wenn man nach dem oben Gesagten die Unumgänglichkeit der Kompression der dünnen Schicht der zweiten Phase in Betracht zieht. So kann man das Durchschneiden eines Eisblockes bei $0^{\circ}$ durch einen mit einem Gewicht versehenen Draht durchaus nicht als eine Bestătigung der Theorie von Le $\mathrm{Ch}$ atelie $\mathrm{r}$ bezeichnen, sondern einfach dadurch erkiären, daB. indem der Draht aufs Eis drückt, er mit derselben Kraft gleichzeitig die zwischen dem unteren Teil des Drahtes und der Oberflăche des Eises hefindliche dünne Wasserschicht komprimiert und $\mathrm{daB}$ die Schmelztempeartur mit dem Druck abnimmt. Die Umänderung eines derartigen Systems erfolgt im Sinne der Gleichung von Clau sius-Clapeyron. Wiederholen wir den geschilderten Versuch mit einem Stück Gold, dessen Oberfläche mit geschmolzenem flüssigen Golde bedeckt ist, so wird derselbe miBlingen, weil im gegebenen Falle mit der Druckzunahme die Schmelztemperatur sich erhöht. Auf dieselbe Weise kann man auch das Zusammenballen des Niederschlages in einer Losung beim Komprimieren mit einem siebartigen Kolben erklären.

Die Theoriedes ungleichförmigen Druckes könnle eine Anwendung auf verschiedene Naturerscheinungen haben, wennes möglich wäre, nurdie eine Phase $z$ u komprimieren, und wenn die sich unterdem Kolben bildende ne ue Phase keine Kompression erleiden, sondern frei den Kolben passieren könnte. Indessen ist die Herstellung eines derartigen, die zweite Phase nicht komprimierenden halbdurchlassigen Kolbens, meiner Meinung uach. nicht nur unmöglich, sondern sogar schwer in der Phantasie vorstellbar. Würden wir nun mit P. N. Pawlow die Theorie des ungleichförmigen Druckes auf die Erscheinungen der Kapillarchemie anwenden wollen, so stellten sich uns noch größere Hindernisse in den Weg, weil wir hierbei z. B. einen Unterschied zwischen den Molekülen der Flïssigkeit und den Molekülen des festen Körpers usw. machen müBten. Auf jeden Fall kann man diese Theorie nur

30) cf. W. Nernst, Le Chatelier. mit der gröbten Anstrengungi" dann anwenden, wenn man (z. B. beim Schmelzen eines festen Körpers) annimmt, daB die Oberflüchenschicht weniger dicht ${ }^{12}$ ) (d. h. weniger orientiert) ist, als die inneren Schichten und quasi ein molekulares Sieb darstellt, durch das die in flüssigen Zustand ubergehenden Molekile von innen herausgedrïckt werden. Mit anderen Worten könnte man die Theorie des ungleichförmigen Drucks nur mit der gröbten Anstrengung zur Erklärung von Erscheinungen bei meiner Vorstellung von der Struktur der Oberfiache disperser fester oder flüssiger Körnchen heranziehen. Nehmen wir aber an, wie es auch gewöhnlich geschieht, $\mathrm{daB}$ die Oberflächenschicht eine elastische und dabei dichtere Membran als die inneren Schichten bildet, so ist die Behauptung, als ob dank einem solchen den Körper umgebenden Panzer derselbe flüchtiger, löslicher und leichtschmelzbarer wird, ebenso absurd, wie die Annahme, dak ein Vogel sich um so leichter aus einem Käfig befreien kann, je dichter die inn umgebenden Drahtmaschen sind.

\section{B. Widerspricht meine Theorie den Tatsachen?}

In 4. seiner Abhandlung versucht $P$. N. Pawlow die Unhaltbarkeit meiner Theorie in bezug auf den Einflub des Dispersitätsgrades auf die physiko-chemischen Eigenschaften zu beweisen. Vor allen Dingen nimmt P. N. Pawlow eine igeschichtliche Korrektion" in meiner Theorie vor und möchte nachweisen, daB ineine Vorstellung von der Beschaffenheit der Flüssigkeitsoberfläche "eine miBglückte Formulierung der bekannten Idee ron van der Waals" ist: ferner weist er zum SchluB des Artikels darauf hin, daB man auf dem schon von der Kapillartheorie heschrittenen und ,von der Willkür des spekulativen Denkens" freien Wege weiterschreiten soll. In diesen Behauptungen kommt noch einmal P. N. Pawlow's ungenügende Kenntnis der Literatur zum Vorschein und um ganz objektiv zu sein, zitiere ich eine diesbezügliche Stelle aus dem Physikkursus von O. D. Chwolson ${ }^{13}$ ): "Eine stichhaltige Erklärung für die Entstehung einer dichten Oberflächen-

11) Auf diese Frage komme ich in dem Buche Kolloidwissenschaft und Struktur der Materie* (Dresden, Theodor Steinkopff), das ich für den Druck vorbereite, noch zurück.

12) Koll-Zeitschr. 7, 207 (1910)

13) 2. Ausgabe, 1, 475 (1900). 
schicht in den Flüs sigkeiten existiert bis jetzt nichtit). Das bloke Vorhandensein in dieser Schicht von nach innen det Flüssigkeit gerichteten Kräften kann nicht als eine Erklärung der Dichte angesehen werden; diese Krăfte müssen einen Druck ausüben, der nach dem Gesetz der Hydrostatik sich in der Plüssigkeit nach allen Richtungen verteilt und ein Dichtwerden der ganzen Masse hervorruft. Einzelne Erwăgungen lassen im Gegenteil eher annehmen, daB die Dichte der Oberflächenschicht geringer ist als die Dichte der übrigen Flüssigkeit . . Besonders G. va n der Men sbrugghe $(1866-1893)$ hat sich mit der Theorie der Oberflächenspannung beschäftigt; er und einige andere Gelehrte nehmen an, dab die Oberflächenschicht der Flüssigkeit keine gröBere, sondern im Gegenteil eine geringere Dichte besitzt als die übrigen Teile der Flüssigkeit.*

Aus diesem Zitat geht klar hervor, dab es bis jetzt noch keine "von der Willkür des spekulativen Denkens" freie Kapillartheorie gibt und dab die "geschichtliche Korrektion ${ }^{15}$ ) von P. N. Paw low vollständig unrichtig ist. Denn wenn er schon überhaupt meine Vorgänger erwăhnt, so hätte er G.van der Mensbrugg he, aber auf keinen Fall van der Waals nennen müssen, weil meine Anschauungen eher mit denen von $G$. van der Mensbrugghe in Zusammenhang gebracht werden könnten, wakrend van der Waals hierbei absolut nicht in Betracht kommt.

Um fernerhin die Unrichtigkeit meiner Anschauungen über die Oberflächenstruktur des Kristalles $2 u$ beweisen, schlăgt P.N. Pawlow in 4. seine gewöhnliche Taktik ${ }^{16}$ ) ein - er legt mir Worte in den Mund, die ich nie

i) Der gesperte Druck stammt von $O$. $D$. Chwolson.

14) Den Leser erinnere ich an eine andere ebensowenig richtige F.W.Kaster betreffende, ge s ch i chtliche Korrektion" von P.N.Pawlow (Koll.-Zeitschr. 7, 206, 1909).

16) Vgl. die Abhandlung von P. N.Pawlow im Journ. d. (uss. chem. Ges. 41, 1779 (1910), in der besonders charakteristisch die Auslegung meiner geschichtlichen Korrektion bezuglich des Schmelzens hiussiger Kornchen (Lord Kelvin) ist; Obwohl schon aus der Ueberschrift dieser Korrektion physikalische und chemische Eigenschaften als Funktionen der Korngrobe fester und flassiger disperser Phasen " deutlich hervorgeht, daB ich die Iropfen als Kornchen der Flinssigkeit bezeichne, und daB sowohl ass dem Text als auch aus der Literaturangabe zweifellos $z$ u ersehen ist, daB von Flussigkeiten die Rede ist, so hat P.N. Pawlow es doch für moglich gehalten, zu behaupten, das ich die Arbeit uber die festen Kornchen Lord Kelvin zuschreibe. (Ueber Dampidruck der kleinen Tropfen, siehe P. N. Pawlow, I. c. 40, 1025 und 1028). gebraucht habe. In meiner Abhandlung '7 schreibe ich deutlich, da $B$ die Oberflachenschicht des Kristalls "natürlich nicht identisch" ist mit einer komprimierten Flüssigkeit; dann schreibe ich: "Die auberordentlich feinen festen dispersen Systeme năhern sich dem flüssigen Zustande, sind aber nicht mit den Flüssigkeiten als solchen in allen Beziehungen identisch"; und schlieblich „eine starke Formschwindung kristallinischer Individuen bei minimal löslichen Körpern ist nur bei überultramikroskopischen Kriställchen zu erwarten ". Mögen die Leser nun selbst entscheiden, ob P. N. P a w low im Recht ist, wenn er meine Anschauungen in folgende Form kleidet: "Eine derartige Auffassung der Kristalloberflächenschicht ist identisch mit der Anerkennung der Is o tropie der Kristalloberflächenschicht." Es ist doch dem Leser bekannt, daB ich die Teilchen in kolloiden Losungen fïr kristallinisch halte. Meine Vorstellung vom geringeren Orientierungsgrad der Kristalloberflăche widerspricht nicht nur nicht den physikalischen und geometrischen Eigenschaften hochdisperser Kristalle, sondern wird sogar völlig durch sie bestätigt.

Aus der Raumgittertheorie, dem Vorhandensein dynamischer Prozesse und einseitiger Wirkung von Molekularkräften auf die Kristalloberfluche geht unmittelbar hervor, dab das Orientierungsvermögen der Moleküle um so mehr gestört wird. je größer der Dispersitätsgrad oder je grötler der dispergierende Einfluk des Dispersionsmittels is ${ }^{\star}$. Mit der Storung des Orientierungsvermōgens nimmt auch die Făhigkeit, die Uebersättigung aufzuheben, ab. Dadurch, daß wir bei verhältnismäßig großen Kristallen durch Auffosen von fremden Molekülen in der Oberflachenschicht den Orientierungsgrad verringern, verkleinern wir auch die Geschwindigkeit der Aufhebung der Uebersăttigung und rufen die Bildung von miBgestalteten, nicht ganz regelmäBig gebildeten Kristallformen hervor. Durch die Arbeiten von O. Lehmann und Marc wird das vollkommen bestătigt.

Meine molekular-kinetische Strukturtheorie der Oberfluche disperser Phasen steht auch nicht mit einer einzigen experimentell festgestellten Erscheinung in Widerspruch und drückt in der Sprache der molekular-kinetischen Theorie nur das aus, was Wilhetm Ostwald (1909) rom energetischea Standpunkt aus in

17) Koll. Zeitschr.6, 32 (1910). 
folgenden Worten zum Ausdruck gebracht hat: . Insofern, als die Oberfläche einer Flüssigkeit mehr Energie enthalt, als diese in der Masse, läbt sich der Oberflachenzustand als eine Zwischenform zwischen der flüssigen und der Dampfform betrachten... Aehnliche Betrachtungen lassen sich für feste Stoffe anstellen, und auch bei diesen wird man mit zunehmender Zerteilung eine Annäherung an die Eigenschaften des flüssigen, bezw. Dampfzustandes erwarten müssen ${ }^{18}$ )."

14) GrundriB, 533, 4. Aufl. 1909.
SchluB.

Aus alfem Angeführter geht, glaube ich, genügend klar hervor, dab die Kritik $P . N$. Pawlow's vollkommen miBlungen ist und leider nicht allein aus rein wissenschaftlichen Motiven hervorgerufen worden ist. Das letztere gibt mir ein Recht. die ferneren Ausfälle von Pawlow's Seite mit Stillschweigen zu übergehen.

\section{St. Petersburg.} den 25. Oktober (7. November) 1910.

\title{
Zur Frage über den Niederschlagsformkoeffizienten von P. P. von Weimarn.
}

\author{
Von P. Pawlow, Odessa.
}

(Eingegangen am 5. Dezember 1910)

1. Chemiker, Kristallographen und Mineralogen interessierten sich von jeher für die Frage nach den Bedingungen, welche die Eigenschaften der aus den Lösungen ausfallenden kristallinischen Phase bestimmen. Die einen interessierten sich für das praktische Problem der Erhaltung von gut gebildeten Kristallen, die anderen wieder betrachteten die Frage über Kristallisationsbedingungen im Zusammenhange mit den intimsten und wesentlichen Problemen der Spezialdisziplinen 1). Unter anderen Fragen über die Kristallisation ist seit langem die Frage über die Bedingungen aufgestelit worden, welche die Korngröbe der sich bildenden kristallinischen Phase bestimmen, indem historisch der Gedankengang in zweierlei Richtung sich entfaltete: Die einen forschten nach Wegen unt Mitteln zur Erhaltung von großen Kristallen ${ }^{2}$ ), die anderen (H. Schiff, G. Buchner, C. Neuberg und W. Neumann, C. Paal und Fr. $K u h n, P$. P. von Weimarn) beschaftigten sich mit der Bildung von feinkristallinischen Systemen.

Angesichts dessen, daß die Frage die Interessen von mehreren Disziplinen berührt, kann man den Versuch P. P. von Weimarn's nicht unberücksichtigt lassen, den Zusammenhang zwischen der Größe des kristallinischen Korns und den Eigenschaften der Lösung, in welcher die kristallinische Phase entsteht, zu ermittein. P. P. von Weimain führt den Be-

1) Die Literatur siehe hei R. Brauns, Chemische Mineralogie.

i) $\mathbf{c}$. griff "des Niederschlagsformkoeffizienten " ein ${ }^{3}$. Dieser Koeffizient ist um so größer, je größer der Dispersitätsgrad des Niederschlags, d. h. je kleiner die Korngrøbe des Niederschlags ist.

Algebraisch wird der Koeffizient der Niederschlagsform so ausgedrückt: $N={ }_{L}^{P}$. Hier ist $\mathrm{N}$ der Niederschlagsformkoeffizient, $\mathrm{P}$ die Bildungsgeschwindigkeit der kristallinischen Phase, $L$ die Konzentration der Loosung über der kristallinischen Phase.

Die Geschwindigkeit der Kristallisation bestimmt P. P. von Weimarn durch die Quantität der Substanz, die in einer Volumeneinheit ausscheidet.

Wenn wir durch $\mathrm{S}$ die Anfangskonzentration der Lösung vor der Kristallisation bezeichnen, so ist $\mathbf{P}=\mathbf{S} \ldots L$. Für $\mathbf{N}$ gibt daher P. $P$. von We im ar $n$ einen andern Ausdruck ${ }^{4}$ ), und zwar

$$
\mathbf{N}=\frac{\mathrm{S}-\mathrm{L}}{\mathrm{L}} \text {. }
$$

Es ist nicht schwer zu ersethen, dab die Formel von P. P. von Weimar $n$ das wirklich zu beobachtende Verbältnis zwischen Lösung und Kristall nicht ausdrückt.

2. Der zweiten Formel kann man die Gestalt $\mathrm{N}=\frac{\mathrm{S}}{\mathrm{L}}-1$ verleihen.

Es ist folglich $\mathrm{N}+\mathrm{I}=\mathrm{S}$

8) Koll.-Zeitschr. 2, 305 (1908).

4) Koll.-Zeitschr. 2, 306 (1908). 